\title{
Whole-genome assembly of Babesia ovata and comparative genomics between closely related pathogens
}

\author{
Junya Yamagishi ${ }^{1,2^{*}} \mathbb{D}$, Masahito Asada ${ }^{3}$, Hassan Hakimi $^{3}$, Takeshi Q. Tanaka ${ }^{4}$, Chihiro Sugimoto ${ }^{1,2}$
} and Shin-ichiro Kawazu ${ }^{5}$

\begin{abstract}
Background: Babesia ovata, belonging to the phylum Apicomplexa, is an infectious parasite of bovids. It is not associated with the manifestation of severe symptoms, in contrast to other types of bovine babesiosis caused by $B$. bovis and B. bigemina; however, upon co-infection with Theileria orientalis, it occasionally induces exacerbated symptoms. Asymptomatic chronic infection in bovines is usually observed only for B. ovata. Comparative genomic analysis could potentially reveal factors involved in these distinguishing characteristics; however, the genomic and molecular basis of these phenotypes remains elusive, especially in B. ovata. From a technical perspective, the current development of a very long read sequencer, MinION, will facilitate the obtainment of highly integrated genome sequences. Therefore, we applied next-generation sequencing to acquire a high-quality genome of the parasite, which provides fundamental information for understanding apicomplexans.
\end{abstract}

Results: The genome was assembled into 14,453,397 bp in size with 5031 protein-coding sequences (91 contigs and N50 = 2,090,503 bp). Gene family analysis revealed that ves 1 alpha and beta, which belong to multigene families in B. bovis, were absent from B. ovata, the same as in B. bigemina. Instead, ves1a and ves 1 b, which were originally specified in B. bigemina, were present. The $B$. ovata and $B$. bigemina ves1a configure one cluster together even though they divided into two sub-clusters according to the spp. In contrast, the ves $1 \mathrm{~b}$ cluster was more dispersed and the overlap among B. ovata and B. bigemina was limited. The observed redundancy and rapid evolution in sequence might reflect the adaptive history of these parasites. Moreover, same candidate genes which potentially involved in the distinct phenotypes were specified by functional analysis. An anamorsin homolog is one of them. The human anamorsin is involved in hematopoiesis and the homolog was present in B. ovata but absent in B. bigemina which causes severe anemia.

Conclusions: Taking these findings together, the differences demonstrated by comparative genomics potentially explain the evolutionary history of these parasites and the differences in their phenotypes. Besides, the draft genome provides fundamental information for further characterization and understanding of these parasites.

Keywords: B. ovata, Babesia, MinlON, Comparative genomics

\footnotetext{
*Correspondence: junya@czc.hokudai.ac.jp

${ }^{1}$ Research Center for Zoonosis Control, Hokkaido University, Sapporo, Japan

${ }^{2}$ Global Station for Zoonosis Control, Gl-CoRE, Hokkaido University, Sapporo,

Hokkaido, Japan

Full list of author information is available at the end of the article
} 


\section{Background}

Babesia ovata is one of the bovine Babesia species originally isolated in Japan [1]. It is transmitted by the ixodid tick, Haemaphysalis longicornis, and is widespread in several East Asian countries [2, 3]. The symptoms that manifest in cattle infected with B. ovata are generally mild, including fever and anemia [4]. Adult animals are more susceptible to such parasitic infection than calves, in whom the infection remains subclinical in most cases. In the chronic stage, the infection is subclinical and the parasitic burden of the infected animal is typically submicroscopic, and can only be detected by more sensitive diagnostics such as PCR $[4,5]$. However, co-infection with Theileria orientalis, which is also transmitted by $H$. longicornis, causes severe anemia and hemoglobinuria $[4,5]$, which then results in a considerable burden to the livestock industry.

Besides B. ovata, several Babesia species are known to be infectious to cattle. $B$. bigemina is the most closely related species to $B$. ovata based on phylogenetic and evolutionary analyses using $18 \mathrm{~S}$ rRNA sequences [6]; however, manifestations are more severe in B. bigemina than in $B$. ovata $[2,7]$. The $B$. bigemina genome is available in public databases and estimated to be $13.8 \mathrm{Mb}$ in size [8]. B. bovis is one of the most extensively investigated Babesia species in terms of both biology and bioinformatics. Indeed, its genome was the earliest to be published among the Babesia spp. [9] and annotation has been improved with EST and RNAseq data $[10,11]$. B. divergens genome sequence is also available [8].

The asexual cycle of Babesia spp. that replicates in erythrocytes is responsible for pathogenesis and clinical symptoms. Bovine babesiosis caused by B. bovis and $B$. bigemina is acute, generally severe, and sometimes lifethreatening [7]. Chronic infection has been reported in $B$. gibsoni and $B$. divergens causing canine and bovine babesiosis, respectively [12, 13], and Theileria spp. [14, $15]$; however, in bovine babesiosis, there is no clear evidence of usual chronic infection, except in cases involving $B$. ovata. Regarding the molecular mechanisms involved in pathogenicity, in cerebral babesiosis caused by $B$. bovis that there is involvement of heterodimeric variant erythrocyte surface antigen (VESA), which is encoded by a multi-gene family, ves $1 \alpha$ and ves $1 \beta[16-$ $20]$. It has also been proposed that differential expression of the ves1 gene is involved in the severity of the manifested symptoms [11]. On the other hand, it has been demonstrated in B. bigemina that their VESA are coded in ves $1 a$ and ves $1 b$, which are distinct from ves $1 \alpha$ and ves $1 \beta$ [8]. B. bigemina also possess the ves 2 gene family, which is distinct from ves1, suggesting diversity and evolutionary dynamics of these gene families [8]. Such diversity potentially explains the difference in pathogenicity among these species. Comparative genomic analysis of other Babesia spp. should provide an overview of the evolutionary dynamics at the genomic level, including in the ves family, which might explain the different characteristics of Babesia spp., such as their pathogenicity. Therefore, we aimed to obtain a draft genome of B. ovata, with the goal of finding the genomic and molecular bases of its unique phenotypes, namely, chronicity, low pathogenicity, and enhanced pathogenicity upon co-infection.

In terms of methods used for genomic analysis, the development of next-generation sequencing (NGS) offers us a powerful tool. In particular, data produced by Illumina platforms provide us with very high-throughput and high-quality sequence reads. The downside of these sequencing technologies is the short length of their reads. They provide $300-\mathrm{bp}$ paired-end data, at most; this leads to misassembly of repetitive regions and multicopy genes. The latest NGS platforms, such as PacBio or MinION, can overcome such disadvantages by generating long reads based on single-molecule sequencing chemistry, although the sequences of these reads are less accurate than those of the Illumina systems. These lessaccurate sequences can be corrected by hybrid assembly, which integrates the two types of NGS, resulting in sets of gene sequences that are of sufficient quality for "omics" data analysis. Since it is known that in silico analysis without experimental data is insufficient to annotate genes and estimate gene models correctly, another hybrid method has been developed to integrate in silico with in vitro/vivo data. AUGUSTUS is one such tool that evaluates gene models based on integration of genome and transcriptome data [21].

In this study, we applied the single-molecule nextgeneration sequencer MinION, together with PacBio RS II expecting high contiguity and accuracy supported by highly reliable Illumina short reads. In brief, 1) reads derived from a MinION and PacBio RS II sequencer were assembled by Canu [22], 2) low-reliability contigs were excluded, and 3) errors were corrected with Pilon using HiSeq reads. In addition, the gene model was estimated based on both information science and an experimental approach supported by RNAseq. Subsequent comparative genomic analyses revealed that the ves $1 a$ and ves $1 b$ multigene family, which was originally identified in $B$. bigemina, also exists in $B$. ovata, while the ves $1 \alpha$ and ves $1 \beta$ multigene family originally identified in $B$. bovis was not present. However, B. bigemina ves $1 b$ did not fully overlap in B. ovata, implying the diversified function of ves $1 b$ in these parasites. In addition, we were able to find $B$. ovata-specific gene families and individual genes, such as extracellular matrix-binding proteins and an anamorsin homolog gene. Their functions remain elusive, but they were identified as potential candidates for having a pivotal function in the pathogenic nature of the parasites. 


\section{Methods}

\section{B. ovata Strain and culture}

The B. ovata Miyake strain was cultured in vitro using purified bovine red blood cells and culture medium M199 supplemented with 40\% bovine serum [23].

\section{Genomic DNA extraction, library construction, and sequencing}

Genomic DNA was extracted from B. ovata-infected RBCs by the standard phenol-chloroform method [24]. The library for MinION was constructed with a Rapid Sequencing Kit, SQK-RAD003 (Oxford Nanopore Technologies), and then analyzed with two FLOMIN106 flow cells. Library construction with a TruSeq DNA PCR-Free Sample Prep Kit (Illumina) and 90-bp paired-end sequencing with HiSeq 2000 (Illumina) were performed at BGI JAPAN [25]. Library construction using Lib_Kit (Pacific Biosciences) and sequencing with PacBio RS II using three P6C4 SMART cells (Pacific Biosciences) were performed at Eurofins MWG Operon, Inc. [25].

\section{RNAseq analysis}

B. ovata total RNA was extracted from infected RBCs, which were cultured in vitro with TRIzol (Sigma), following the manufacturer's instructions. Quality and quantity of the purified RNA were validated with Bioanalyzer (Agilent). Library construction for RNAseq was performed as per the instruction manual with TruSeq Stranded mRNA LT Sample Prep Kit (Illumina), and the product was subjected to HiSeq 2500 (Illumina) with the 101-bp paired-end protocol (Illumina).

\section{De novo genome assembly}

Reads obtained from MinION and PacBio RS II were assembled with Canu using genome size $=14 \mathrm{~m}$ and default settings for the other parameters [22]. The resulting contigs were examined to subtract possible host contamination and artifacts based on the following criteria. The first criterion was evaluated by relative coding capacity between bovine and Babesia parasites ( $B$. bigemina and $B$. bovis) evaluated by BLASTX, and coverage less than 10 (mean and standard deviation in the top 10 longest contigs were 29.1 and 4.1, respectively) or if the best hit was against bovine. The second criterion was performed using redundancy among all contigs. The ratio among sequences with similarity to the other contigs evaluated by BLASTN and the whole length in each contig were calculated. The contig with the highest ratio was excluded by iterative analysis until the ratio became less than $90 \%$. The qualified contigs were polished using Illumina reads with Pilon with 26 iterations until a plateau was reached [26].

\section{Gene model estimation and functional annotation}

For gene model estimation, we applied AUGUSTUS version 3.1.0 [21]. To establish trained parameters for AUGUSTUS, webAugustus [27] was utilized, and the required data, the genome sequence (PiroplasmaDB5.1_BbovisT2Bo_Genome.fasta), and the full-length EST (B.bov.FL-EST.fa) for B. bovis were obtained from PiroplasmaDB and DB-AT, respectively [28, 29]. The sequences derived from the RNAseq analysis were mapped onto the assembled draft genome sequence of B. ovata with Tophat2. Paired-end reads that failed to be mapped were subtracted by SAMtools. AUGUSTUS in step 1 was performed as follows. An intron hints file with gff format was created by bam2hints script using the Bam file originating from the mapping step. The first AUGUSTUS pass was performed with the trained parameter based on $B$. bovis, the hints gff file, and a parameter file, extrinsic.M.RM.E.W.cfg, which was bundled in the package. The results and hint gff were integrated to obtain an intron dataset and converted to obtain an exonexon junction database (exex.fa and map.psl) by intron2exex.100.pl script modified from the original intron2exex.pl to accept 100-bp reads. AUGUSTUS analysis in step 2 was performed as follows. The MiSeq RNAseq reads were mapped to the exon-exon junction database with Bowtie 2 to obtain spliced reads and their mapped profiles. Unmapped reads were discarded with SAMtools and then the remaining sequences were formatted with SAMmap.pl script with reference to map.psl. The mapped results of the MiSeq reads on B. ovata were further filtered to remove $\mathrm{N}$ nucleotides with BamTools with operation $\mathrm{N}$ filter.txt script in the AUGUSTUS package. The filtered and mapped results were merged with those for spliced reads annotated as above. Another intron file, hints 2, was generated from the merged mapped profile and then a second AUGUSTUS implementation was performed with the trained parameter, hints 2, and extrinsic.M.RM.E.W.cfg.

Functional annotation of B. ovata genes, including GO terms, was conducted by Blast2GO [30]. tRNA and rRNA genes in the genome were predicted by tRNAscan [31] and RNAmmer 1.2 web servers [32] with default parameters, respectively.

\section{Comparative genomics}

For the comparative genomic analysis among apicomplexan parasites, we used genome assembly and annotation released in PiroplasmaDB-5.1 for the B. bovis T2Bo strain and the B. microti RI strain, ToxoDB-27 for the $T$. gondii ME49 strain, PlasmoDB-13.0 for the P. falciparum 3D7 strain, and GCA_000981445.1 in BioProject PRJEB5046 for the $B$. bigemina BOND strain.

The coding capacities for both tRNA and rRNA in $B$. bigemina, B. bovis, B. microti, P. falciparum, and T. gondii 
were estimated based on the genome sequences by the same method as used in B. ovata. Homologs among $B$. ovata, B. bigemina, B. bovis, B. microti, P. falciparum, and $T$. gondii were specified by OMA version 2.1.1 with the default parameters [33]. To examine weak similarity, amino acid sequences coded in $B$. ovata were aligned with those in B. bigemina, B. bovis, B. microti, P. falciparum, and T. gondii using BLASTP with a threshold of more than $30 \%$ identity in more than $30 \%$ of the region of the query sequence. To describe the overall relationship among genes in $B$. ovata, B. bigemina, and B. bovis, an all vs. all BLASTP homology search among them was performed, and gene pairs reciprocally sharing 60\% identity over 150 amino acids were selected and then visualized with Gephi (open-source software for exploring and manipulating networks) using a Fruchterman-Reingold layout. Clusters were also specified by Gephi and their representative annotations were referenced from GCA_000981445.1_Bbig001_genomic.gff for B. bigemina provided by NCBI and PiroplasmaDB-5.1_BbovisT2Bo.gff for B. bovis provided by PiroplasmaDB. The annotation for VESA was acquired from the FTP site provided by the Wellcome Trust Sanger Institute. Reordering of the $B$. ovata assemblies along with $B$. bigemina was performed with Mauve [34]. A dot-plot among the reordered contigs and B. bigemina genome was described using YASS [35].

\section{Expression analysis}

Sequence reads from parasite RNA were also examined to obtain expression profiles. These were mapped onto the draft genome sequence along with the predicted gene model with Tophat 2 [36]. The information obtained was further mapped to the gene model with HTseq [37] to establish the number of reads covering each gene.

\section{Results and discussion}

\section{De novo assembly of $B$. ovata genome}

A total of 459,000 reads and 389,000 subreads, consisting of 1.6 and $0.9 \mathrm{Gbp}$, were obtained using MinION FLO-MIN106 and PacBio RS II, respectively (Table 1).
In parallel, 12.4 million paired-end reads, consisting of 2.2 Gbp, were obtained using MiSeq.

With Canu assembler, these reads were assembled into 228 contigs comprising approximately 16.2 Mbp. Following subtraction based on redundancy, possible host contamination, and coverage, 91 contigs were qualified (Table 1). The Cane assembler improves the accuracy of the resulting sequences by making consensus in each base; however, it is known that there remain some sequencing errors after this operation. Therefore, we conducted error correction of the HiSeq-derived reads using Pilon. Substitution of $1368 \mathrm{nu}$ cleotides, 24,737 insertions and 783 deletions were corrected accordingly, and then the draft genome consisting of $14,453,397$ bp was successfully obtained (Table 1 ). The longest contigs, $\mathrm{N}_{50}$, and $\mathrm{nN}_{50}$ were $3,702,329$ bp, $2,090,503 \mathrm{bp}$, and the third longest contig, respectively, suggesting this is a draft but nearly complete genome with high contiguity. In this study, we applied MinION sequencer to apicomplexan parasites for the first time. Both MinION and Pac Bio sequencers are known to read out long sequences, which facilitates high-contiguity genome assembly $[38,39]$. In particular, it is known that MinION produces ultralong sequences. Indeed, we obtained a 93,434-bp-long read at most in this study and $\mathrm{N}_{50}$ was improved from 97,655 by Pac Bio reads only to $2,093,449$ by MinION and Pac Bio reads together or 408,732 by MinION only. This suggests the utility of MinION in the assembly of genomes with complicated structures, such as the multiple family genes in apicomplexan parasites.

A genome sequence with high contiguity also provides an opportunity for synteny analysis. We aligned the $B$. ovata draft genome sequence on the $B$. bigemina genome and found that structures of chromosomes I, IV, and V in $B$. bigemina were well conserved in B. ovata. In contrast, chromosomes II and III were rearranged among them. In particular, more rearrangements were observed between chromosome II in B. bigemina and the longest contig of B. ovata (Fig. 1). This information is useful for future analysis of the evolutionary history among related species.

Table 1 Statistics of sequenced reads and contigs

\begin{tabular}{lllll}
\hline Assembly method & Canu & Canu & Canu & HGAP3 \\
Subtraction of unreliable contigs & Yes & No & No & $16,481,566$ \\
total base pairs & $14,453,397$ & $16,393,820$ & $228,312,816$ & 206 \\
\# of contigs & 91 & $2,093,449$ & 408,732 & 97,655 \\
N50 contig length & $2,090,503$ & 3 & 11 & 48 \\
N50 contig rank & 3 & $3,692,619$ & $1,286,507$ & 529,343 \\
max contig length & $3,702,329$ & 458,947 & 458,947 & 0 \\
\# of MinlON raw reads & 458,947 & $1,592,957,533$ & $1,592,957,533$ & 0 \\
\# of MinlON total base pairs & $1,592,957,533$ & 388,599 & 0 & 388,599 \\
\# of PacBio sub reads & 388,599 & $954,792,480$ & 0 & $954,792,480$ \\
\# of PacBio total base pairs & $954,792,480$ & &
\end{tabular}




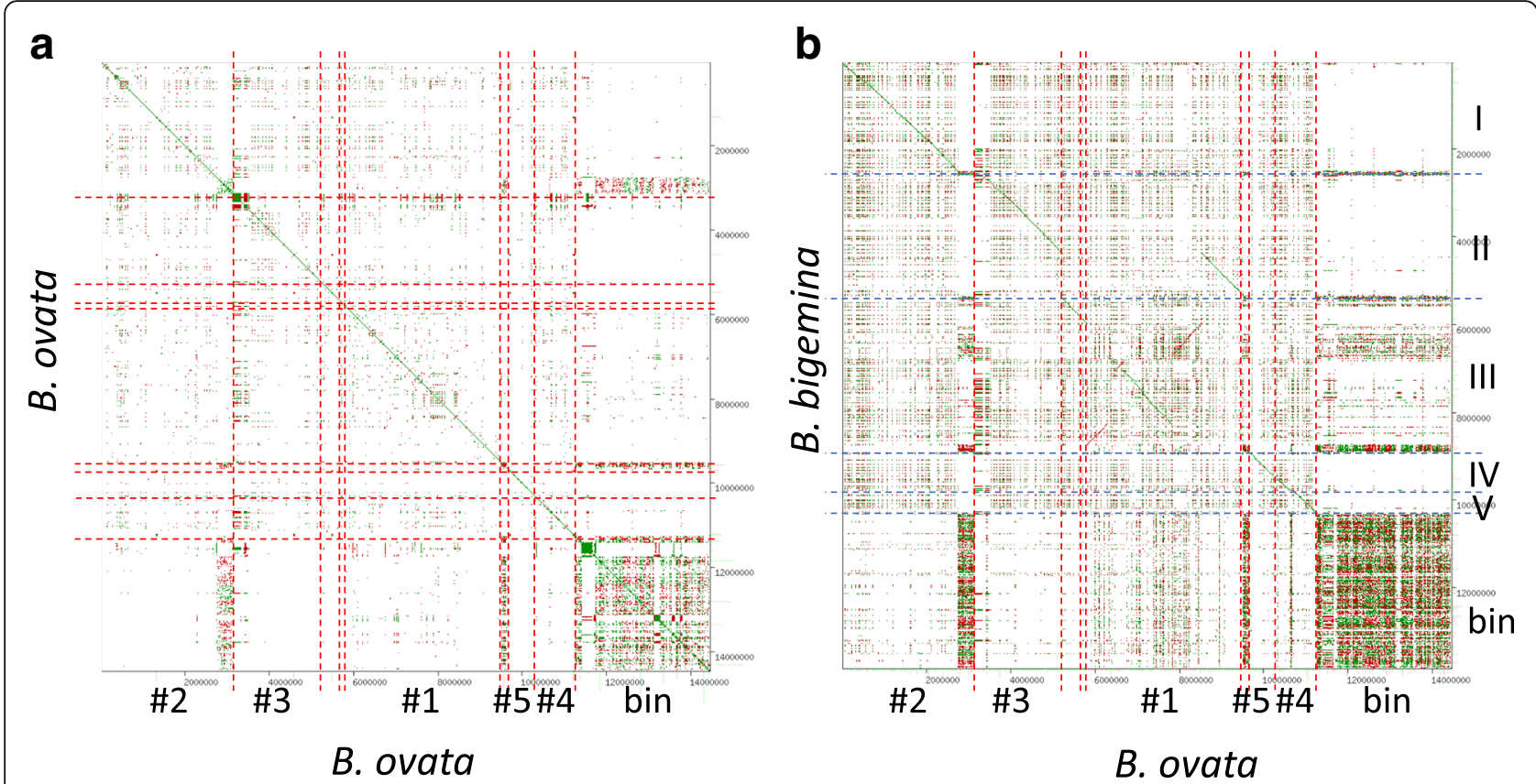

Fig. 1 Homology and synteny analysis by dot-plot analysis. Dot-plot comparisons of B. ovata against itself (a) and B. ovata vs. B. bigemina (b) are presented. The vertical and horizontal dotted lines separate each contig. The Roman numerals on the right side represent the names of contigs in B. bigemina. The bin is not a contig but a bundle of short contigs. ID for major contigs in B. ovata are also represented. The orders are available in Additional file 4: Table S3

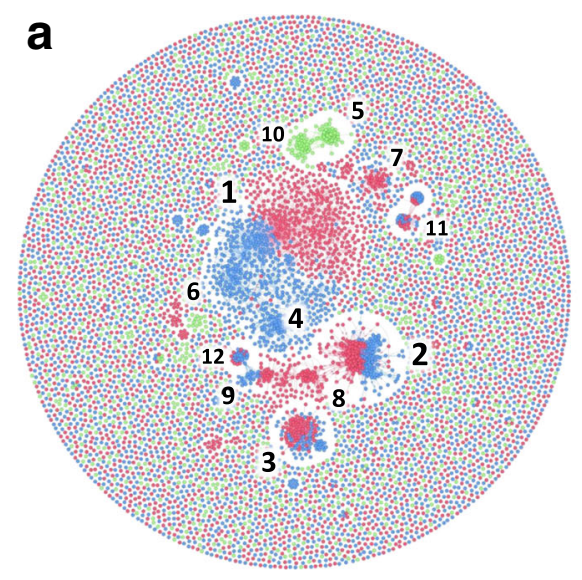

A $\mid \square$ B. ovata

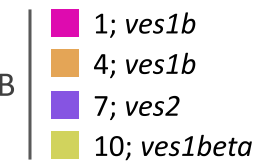

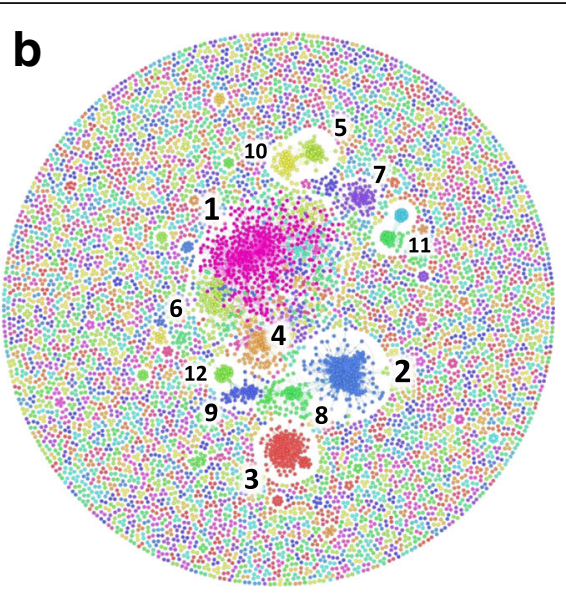

B. bigemiina

2; ves1a

5; ves1alpha

8; ebh, putative

11; $m f s m p$
B. bovis

3; hypothetical

6; ves1b

9; ves1ba

12; reverse transcriptase

Fig. 2 Homolog clustering based on sequence similarity of all genes in B. ovata, B. bigemina, and B. bovis. Each node represents a protein-coding gene in the three parasites. Edges represent similarity between connected nodes. Numbers represent representative cluster ID. a The red, green, and blue nodes represent genes in B. ovata, B. bigemina, and B. bovis, respectively. b Differences in color correspond to each cluster 


\section{Gene model estimation}

For gene prediction, tools based on hidden Markov models such as Glimmer and Genscan are commonly utilized after de novo genome assembly [40, 41]. However, information obtained from such genome sequences is not sufficient to predict authentic gene models and occasionally results in incorrect annotation. Hybrid methods with experimental data are efficacious to avoid such incorrect annotations. In this study, we also applied an additional hybrid strategy, supported by transcriptomics analysis with AUGUSTUS, a tool enabling hybrid annotation with genome and transcriptome data in order to obtain more reliable gene models with functional annotations. The transcriptome of B. ovata was obtained from cultured cells by RNAseq using HiSeq 101 paired-end reads. A total of $23.5 \mathrm{Mbp}$ pairedend reads were obtained, which were then mapped on the genome; $60.9 \%$ of these were successfully mapped with Tophat 2 and the remaining reads were assumed to be hostderived reads or the result of unpaired read mapping. Based on the mapping information, AUGUSTUS predicted 5031 coding regions (CDS), which is almost equal to that for $B$. bigemina (Table 2). In addition, tRNAs and rRNAs were identified with tRNAscan [42] and RNAmmer, respectively, with 64 tRNAs encompassing 20 amino acids, six 5S rRNAs, three $18 \mathrm{~S}$ rRNAs, and four $28 \mathrm{~S}$ rRNAs, which is consistent with the most phylogenetically closely related Babesia spp., B. bigemina, and B. bovis (Table 2).

\section{Functional annotation}

Functional identification of the predicted genes was performed with Blast2GO. This provided putative annotation, Gene Ontology (GO), Enzyme Commission (EC) numbers, and Inter Pro Scan results (Additional file 1: Table S1). Based on the analysis, 1371 genes were predicted as hypothetical proteins, whereas the remaining 3660 genes were functionally annotated. In addition, GO and EC identities were assigned to 4305 and 587 genes, respectively. This information was included in a gff file (Additional file 2: Bovata.genome.7.1.gff3).

\section{Comparative genomics}

Next, we performed comparative genomic analysis among typical apicomplexan parasites, B. bigemina, $B$. bovis, B. microti, P. falciparum, and T. gondii, based on amino acid similarity using OMA and BLASTP (Table 2 ). Both sets of genome-wide results suggested that $B$. ovata and $B$. bigemina were the most closely related species, which is consistent with previous studies based on individual genes [2, 43].

Subsequently, we focused on multiplexed gene families among B. ovata, B. bigemina, and B. bovis, such as the variant erythrocyte surface antigen (VESA). The VESA protein of $B$. bovis is encoded by multiple copy ves $1 \alpha$, ves $1 \beta$, and much shorter ves 2 family genes. The heterodimer of VESA 1a and VESA $1 \mathrm{~b}$ is responsible for antigenic variation of the parasite $[19,44]$. Moreover, B. bovis VESA is proposed to be involved in cerebral babesiosis via its ability to adhere to host endothelial cells [20]. Comparative analysis among $B$. bovis strains also suggested that VESA is related to the pathogenicity of the parasite [11]. In our study, B. bovis ves $1 \alpha$ and ves $1 \beta$ were also clearly differentiated into clusters \#5 and \#10, respectively (Fig. 2b, Additional file 3: Table S2). These clusters were $B$. bovis-specific and neither $B$. ovata nor $B$. bigemina genes were included in them, consistent with a previous study of the $B$. bigemina genome [8]. Instead of ves $1 \alpha$ and ves $1 \beta$ forming VESA, it is thought that $B$. bigemina has ves $1 a$ and ves $1 b$ repertoires [8]. Regarding $B$. bigemina ves1a, this is featured in a cluster $(\# 2)$ together with $B$. ovata genes, suggesting that the $B$. ovata genes were ves1a (Fig. 2, Additional file 3: Table S2). However, in the detailed analysis, B. ovata genes and B. bigemina genes were discriminated into two sub-clusters, suggesting that they were not simple ortholog pairs between B. ovata and B. bigemina (Fig. 2c). This implies that

Table 2 comparative analysis of genome, gene and homology among representative apicomplexan parasites

\begin{tabular}{|c|c|c|c|c|c|c|}
\hline & B. ovata & B. bigemina ${ }^{b}$ & B. bovis ${ }^{\mathrm{C}}$ & B. microti & P. falciparum ${ }^{\mathrm{e}}$ & T. gondiif \\
\hline Genome size (bp) & $14,453,397$ & $13,840,936$ & $8,179,706$ & $6,392,438$ & $23,332,831$ & $65,668,596$ \\
\hline \# of coding genes & 5031 & 5079 & 3706 & 3494 & 5542 & 8322 \\
\hline \# of tRNA & 64 & 46 & 69 & 44 & 76 & 184 \\
\hline \# of $5 \mathrm{~S}$ rRNA & 6 & 6 & 9 & 2 & 3 & 47 \\
\hline \# of $18 \mathrm{~S}$ rRNA & 3 & 3 & 3 & 2 & 5 & 44 \\
\hline \# of $28 \mathrm{~S}$ rRNA & 4 & 3 & 3 & 2 & 6 & 32 \\
\hline \# of B. ovata ortholog (OMA) & $\mathrm{N} / \mathrm{A}$ & 3142 & 2312 & 1243 & 819 & 693 \\
\hline \# of B. ovata homologs (blastp) & N/A & 4311 & 3084 & 1856 & 1630 & 1836 \\
\hline
\end{tabular}

${ }^{\mathrm{a} D D B J}$ accession number BDSA01000001-01000091

${ }^{b}$ GenBank assembly accession number GCA 000981445.1

${ }^{\mathrm{C}}$ Registration in PiroplasmaDB reserase 5.1

${ }^{\mathrm{d}}$ Registration in PiroplasmaDB reserase 5.1

${ }^{\mathrm{e}}$ Registration in PlasmoDB reserase 13.0

${ }^{\mathrm{f}}$ Registration in ToxoDB reserase 27 
orthologs from the common ancestor multiplied independently in each ancestral parasite lineage over the course of evolutionary history, as suggested previously [8], which might be a process that is still underway. Regarding ves $1 b$, there were three clusters containing $B$. bigemina ves $1 b$ (\#1, $\# 4$, and \#6). Among them, \#4 and \#6 were B. bigemina-specific (Fig. 2a and b). In contrast, \#1 consisted of both $B$. ovata genes and B. bigemina genes (Fig. 2a and b). Namely, $B$. bigemina had more diversified ves $1 b$ genes than $B$. ovata. Besides, cluster \#1 was also discriminated into sub-clusters corresponding to $B$. ovata genes and B. bigemina genes, the same as cluster \#2 for ves1a (Fig. 2d). The other VESA-like gene family, ves2, is specified in B. bigemina [8]. This lacks the $\mathrm{C}$-terminal transmembrane motif and GPI anchor signal [8]. In B. bigemina, 116 genes were assigned as ves2, and 18 of these were included in cluster \#7 together with 42 B. ovata genes (Fig. 2, Additional file 3: Table S2). Other $B$. bigemina ves 2 formed clusters \#24, \#31, and \#32 and they were almost all $B$. bigemina-specific (Fig. 2, Additional file 3: Table S2). In contrast, B. ovata exhibited specific gene clusters, such as \#8 involving extracellular matrix-binding protein genes $(e b h), \# 13$ with extracellular matrix-binding proteins including spectrin repeats, and \#15 for an additional $e b h$ (Fig. 2, Additional file 3: Table S2). Most of the $e b h$ genes were predicted to have transmembrane domains and metal ion-binding proteins (GO: 0046872) (Fig. 2, Additional file 1: Table S1 and Additional file 3: Table S2).

In addition, we observed multiplication of six-cysteine (6-Cys) domain-containing proteins (IPR010884) unique to $B$. ovata and B. bigemina and corresponding to cluster \#3 (Fig. 2, Additional file 1: Table S1 and Additional file 3: Table S2). Most of them possessed signal peptides, implying that they were secreted proteins. In B. bovis, 10 6-Cys domain-containing proteins were identified and predicted to be either secreted or surface membrane-bound and whose expression may be stage-specific $[45,46]$; however, cluster \#3 and the B. bovis 6-Cys genes did not overlap with each other. In Plasmodium, sexual stage-specific surface antigen $P f s 48 / 45$ is also a known 6-Cys protein [47]; however, none of the Babesia 6-Cys genes showed homology to $P f s 48 / 45$. As observed here, most of the specified gene families were related to cell-surface expression. It was not surprising to predict that surface proteins are involved in pathogenicity in general. Indeed, it is reported that VESA, PfEMP1, and VSG, as major antigens involved in the immune evasion of Babesia, Plasmodium, and Trypanosoma, respectively, are involved in this process $[11,48,49]$. In this analysis, we observed that ves1a and ves $1 b$ were shared between $B$. ovata and B. bigemina, but divided into subclusters. This was more pronounced in the case of ves $1 b$. B. ovata-specific gene amplification was also observed in $e b h$ and others. The acquired diversity potentially explains the differences in phenotype, including in pathogenicity.
In parallel, we also focused on individual unique genes encoded in the B. ovata genome; 1788 and 420 genes have no orthologs and showed no similarity to any of the representative apicomplexan parasites, based on OMA- and Blast-based analyses, respectively (Additional file 1: Table S1). For most of these B. ovata-specific genes, the function was unclear, except for some such as a GCN5-related Nacetyltransferase (GNAT) family protein (BOVATA_003400) (Additional file 1: Table S1). The GNAT family proteins are known to have histone acetyltransferase (HAT) activity [50]. Those identified in T. gondii and $P$. falciparum, TgMYST and PfMYST, have also been demonstrated to exhibit HAT activity and to be involved in gene expression $[50,51]$. These genes are functional homologs of BOVATA_003400; however, they did not exhibit significant sequence similarity. Hence, this gene might be associated with critical functions of the parasites via transcriptional regulation or degradation of effector chemicals created by their hosts. tRNA-dihydrouridine synthase (BOVATA_012420) and thioredoxin domain-containing proteins (BOVATA_013620) were identified as other $B$. ovata-specific genes, for which there are functional homologs in other apicomplexan parasites; however, no significant sequence similarity was identified. Therefore, their functional involvement in phenotypes is much more elusive.

The other category of distinct genes in B. ovata involves those that are conserved across the apicomplexan species, except in B. bigemina (Additional file 1: Table S1). The associated symptoms are among the clear differences between $B$. ovata and B. bigemina, and these $B$. ovata-specific genes have the potential to explain such differences. For example, the anamorsin homolog (BOVATA_026810) belongs to this category. Human anamorsin is known to be involved in the suppression of apoptosis in hematopoietic cells $[52,53]$. Hence, the homolog in B. ovata may also prevent the degradation of hematopoietic cells by the suppression of apoptosis. In contrast, upon $B$. bigemina infection, which lacks such a homolog, severe anemia might result. Mitochondrial import inner membrane translocase subunit (BOVATA_040550) and subtilisin-like protease (BOVATA_022090) also belong to the same category; however, associations among the genes and the differences in phenotype remain elusive.

\section{Transcriptome of $B$. ovata}

Based on RNAseq reads, we obtained transcript frequency data for B. ovata (Additional file 1: Table S1). Actin (BOVATA_041600) and ribosomal-related proteins (BOVATA_006170, BOVATA_029000, BOVATA_030990, and BOVATA_020930) were included in a group of highly transcribed genes, as expected. Some highly transcribed $B$. ovata-specific genes were also observed, like hypothetical protein (BOVATA_015540) and methyltetrahydrofolate- 
homocysteine methyltransferase (BOVATA_026400). These proteins could be potential targets as diagnostic antigens. Besides, highly transcribed putative merozoite surface glycoproteins (BOVATA_028710 and BOVATA_028720) might be potential targets as vaccines. Additionally, BOVATA_021460, which has an ap2 domain most similar to that of Plasmodium AP2-G and is highly similar (87\% amino acid identity over the entire sequence) to the $B$. bigemina AP2 gene (BBBOND_0104820), was highly transcribed. This implies a potential role of the gene in stagespecific transcription, as reported in other Plasmodium parasites $[54,55]$. Future comparative transcriptomics in vivo and in vitro, with or without $T$. orientalis co-infection, and investigation of intermediate stages, such as acute and chronic infection in ticks, should provide a deeper understanding of babesiosis.

\section{Conclusions}

In this study, we succeeded in obtaining a nearly complete genome, established gene models, and conducted functional annotation by integrating three NGS platforms, MinION, PacBio RS II, and Miseq. We also developed hybrid gene model annotations with genomic and transcriptomic data. By performing comparative genome analysis, we found limited diversity in ves $1 b$ and $B$. ovata-specific expansion of $e b h$ genes, together with a number of $B$. ovata-specific genes such as an anamorsin homolog, which is potentially involved in hematopoiesis in infected hosts. We suggest that the involvement of these genes in the unique phenotypes of $B$. ovata should not be overlooked, even though in the future, these candidates must be examined to verify our hypothesis, taking advantage of the available gene manipulation tools for this parasite [56].

\section{Additional files}

Additional file 1: Table S1. Functional annotation of B. ovata genes. Seq_name: Name of amino acid sequence corresponding to annotated genes. RPKM: Normalized mapped RNAseq reads. Columns K to O: Gene ID of orthologs identified with OMA. Columns P to T: Existence of homologs in each species. 1 and 0 represent with and without homologs, respectively. Regarding the other terms in the header, see the Blast2GO publication. (XLSX $1037 \mathrm{~kb}$ )

Additional file 2: Annotation with gff format. (GFF3 $3895 \mathrm{~kb}$ )

Additional file 3: Table S2. Homology clustering. Cluster ID: Cluster ID. Gene: Genes constituting the cluster. Cluster size: Number of genes in the cluster. Annotation: Annotation of the gene. VESA: Appendix annotation for VESA genes. (XLSX $438 \mathrm{~kb}$ )

Additional file 4: Table S3. List of contig ID aligned with B. bigemina genome. (TXT $1 \mathrm{~kb}$ )

\section{Abbreviations}

CA: Celera assembler; CDS: Coding region; ebh: Extracellular matrix-binding protein genes; EC: Enzyme Commission number; EST: Expressed sequence tag; GNAT: GCN5-related N-acetyltransferase; GO: Gene Ontology; NGS: Nextgeneration sequencing; VESA: Variant erythrocyte surface antigen

\section{Acknowledgments}

We thank Aiko Ohnuma for conducting sequencing.

\section{Funding}

This research was supported by a grant from the Science and Technology Research Promotion Program for Agriculture, Forestry, Fisheries and Food Industry to SK, MA, and JY (25018A).

\section{Availability of data and materials}

The genome sequence and annotation are available at DNA Data Bank of Japan (DDBJ; http://www.ddbj.nig.ac.jp) under accession numbers BDSA01000001-BDSA0100091. All in-house scripts applied in this study are available upon request from the corresponding author.

\section{Authors' contributions}

$J Y, C S$, and SK conceived and designed the study. MA, HH, and $\Pi$ conducted sample collection. MA, HH, and TT performed the experiments. $J Y, M A$, and $\mathrm{HH}$ conducted the literature search, performed data extraction and analysis, and interpreted the results. JY drafted and wrote the manuscript. MA, HH, TT, CS, and SK critically reviewed the manuscript for important intellectual content and revised the manuscript. All authors read and approved the final manuscript.

Ethics approval and consent to participate

Not applicable.

\section{Consent for publication}

Not applicable.

\section{Competing interests}

The authors declare that they have no competing interests.

\section{Publisher's Note}

Springer Nature remains neutral with regard to jurisdictional claims in published maps and institutional affiliations.

\section{Author details}

${ }^{1}$ Research Center for Zoonosis Control, Hokkaido University, Sapporo, Japan. ${ }^{2}$ Global Station for Zoonosis Control, GI-CoRE, Hokkaido University, Sapporo, Hokkaido, Japan. ${ }^{3}$ Institute of Tropical Medicine, Nagasaki University,

Nagasaki, Japan. ${ }^{4}$ Faculty of Medicine, Kagawa University, Takamatsu, Japan. ${ }^{5}$ National Research Center for Protozoan Diseases, Obihiro University of Agriculture and Veterinary Medicine, Obihiro, Japan.

Received: 9 May 2017 Accepted: 20 October 2017

Published online: 27 October 2017

\section{References}

1. Minami T, Ishihara T. Babesia ovata sp.n. isolated from cattle in Japan. Nat Inst Anim Health Q. 1980;20:101-13.

2. Sivakumar T, Igarashi I, Yokoyama N. Babesia ovata: taxonomy, phylogeny and epidemiology. Vet Parasitol. Netherlands. 2016;229:99-106.

3. Yoshinari T, Sivakumar T, Asada M, Battsetseg B, Huang X, Lan DTB, et al. A PCR based survey of Babesia ovata in cattle from various. 2013:75:211-4

4. Fujinaga T. Bovine babesiosis in Japan: clinical and clinico-pathological studies on cattle experimentally infected with Babesia ovata. Nihon Juigaku Zasshi. Japan. 1981;43:803-13.

5. Sivakumar $T$, Tagawa M, Yoshinari $T$, Ybanez AP, Igarashi I, Ikehara $Y$, et al. PCR detection of Babesia ovata from cattle reared in Japan and clinical significance of coinfection with Theileria orientalis. J Clin Microbiol. United States. 2012;50:2111-3.

6. Lack JB, Reichard MV, Van Den Bussche RA. Phylogeny and evolution of the Piroplasmida as inferred from $18 \mathrm{~S}$ rRNA sequences. Int J Parasitol. England. 2012;42:353-63.

7. Bock R, Jackson L, de Vos A, Jorgensen W. Babesiosis of cattle. Parasitology. 2004;129(Suppl):S247-69.

8. Jackson AP, Otto TD, Darby A, Ramaprasad A, Xia D, Echaide IE, et al. The evolutionary dynamics of variant antigen genes in Babesia reveal a history of genomic innovation underlying host-parasite interaction. Nucleic Acids Res. England. 2014;42:7113-31. 
9. Brayton K a, Lau AOT, Herndon DR, Hannick L, Kappmeyer LS, Berens SJ, et al. Genome sequence of Babesia bovis and comparative analysis of apicomplexan hemoprotozoa. PLoS Pathog. 2007;3:1401-13.

10. Yamagishi J, Wakaguri H, Yokoyama N, Yamashita R, Suzuki Y, Xuan X, et al. The Babesia bovis gene and promoter model: an update from full-length EST analysis. BMC Genomics. England. 2014;15:678.

11. Pedroni MJ, Sondgeroth KS, Gallego-Lopez GM, Echaide I, Lau AOT. Comparative transcriptome analysis of geographically distinct virulent and attenuated Babesia bovis strains reveals similar gene expression changes through attenuation. BMC Genomics. England. 2013;14:763.

12. Wozniak EJ, Barr BC, Thomford JW, Yamane I, McDonough SP, Moore PF, et al. Clinical, anatomic, and immunopathologic characterization of Babesia gibsoni infection in the domestic dog (Canis familiaris). J Parasitol. 1997;83:692-9.

13. Moreau E, Jouglin M, Chauvin A, Malandrin L. Babesia divergens experimental infection of spleen-intact sheep results in long-lasting parasitemia despite a strong humoral response: preliminary results. Vet Parasitol. 2009;166:205-11.

14. Skilton RA, Bishop RP, Katende JM, Mwaura S, Morzaria SP. The persistence of Theileria parva infection in cattle immunized using two stocks which differ in their ability to induce a carrier state: analysis using a novel blood spot PCR assay. Parasitology. 2002;124:265-76.

15. Dobbelaere DA, Fernandez PC, Heussler VT. Theileria parva: taking control of host cell proliferation and survival mechanisms. Cell Microbiol. 2000;2:91-9.

16. Allred DR, Carlton JM, Satcher RL, Long JA, Brown WC, Patterson PE, et al. The ves multigene family of $B$. bovis encodes components of rapid antigenic variation at the infected erythrocyte surface. Mol Cell. 2000;5:153-62.

17. Al-Khedery B, Allred DR. Antigenic variation in Babesia bovis occurs through segmental gene conversion of the ves multigene family, within a bidirectional locus of active transcription. Mol Microbiol. 2006;59:402-14.

18. Sondgeroth KS, McElwain TF, Allen AJ, Chen A V, Lau AOT. Loss of neurovirulence is associated with reduction of cerebral capillary sequestration during acute Babesia bovis infection. Parasit. Vectors. 2013;6:181

19. Xiao Y-P, Al-Khedery B, Allred DR. The Babesia bovis VESA1 virulence factor subunit $1 \mathrm{~b}$ is encoded by the 1 beta branch of the ves multigene family. Mol Biochem Parasitol. 2010;171:81-8.

20. O'Connor RM, Allred DR. Selection of Babesia bovis-infected erythrocytes for adhesion to endothelial cells. coselects for altered variant erythrocyte surface antigen isoforms. J Immunol. 2000;164:2037-45.

21. Stanke M, Diekhans M, Baertsch R, Haussler D. Using native and syntenically mapped cDNA alignments to improve de novo gene finding. Bioinformatics. England. 2008;24:637-44.

22. Koren S, Walenz BP, Berlin K, Miller JR, Bergman NH, Phillippy AM. Canu: scalable and accurate long-read assembly via adaptive k-mer weighting and repeat separation. Genome Res. 2017;27:722-36.

23. Igarashi I, Avarzed A, Tanaka T, Inoue N, Ito M, Omata Y, et al. Continuous in vitro cultivation of Babesia ovata. J Protozool Res. 1994:4:111-8.

24. Green MR, Sambrook J. Molecular cloning: a laboratory manual. Fourth ed. 2012.

25. Liu L, Li Y, Li S, Hu N, He Y, Pong R, et al. Comparison of next-generation sequencing systems. J Biomed. Biotechnol. 2012;2012:251364.

26. Walker BJ, Abeel T, Shea T, Priest M, Abouelliel A, Sakthikumar S, et al. Pilon: an integrated tool for comprehensive microbial variant detection and genome assembly improvement. Wang J, editor. PLoS One 2014;9:e112963.

27. Hoff KJ, Stanke M. WebAUGUSTUS-a web service for training AUGUSTUS and predicting genes in eukaryotes. Nucleic Acids Res. England. 2013;41:W123-8.

28. Ma C, Gunther S, Cooke B, Coppel RL. Geneious plugins for the access of PlasmoDB and PiroplasmaDB databases. Parasitol Int. Netherlands. 2013;62:134-6.

29. Jakalski M, Wakaguri H, Kischka TG, Nishikawa Y, Kawazu S, Matsubayashi M, et al. DB-AT: a 2015 update to the full-parasites database brings a multitude of new transcriptomic data for apicomplexan parasites. Nucleic Acids Res. England. 2015;43:D631-6.

30. Conesa A, Gotz S, Garcia-Gomez JM, Terol J, Talon M, Robles M. Blast2GO: a universal tool for annotation, visualization and analysis in functional genomics research. Bioinformatics. England. 2005;21:3674-6.

31. Schattner P, Brooks AN, Lowe TM. The tRNAscan-SE, snoscan and snoGPS web servers for the detection of tRNAs and snoRNAs. Nucleic Acids Res. England. 2005;33:W686-9.

32. Lagesen $\mathrm{K}$, Hallin $\mathrm{P}$, Rodland EA, Staerfeldt H-H, Rognes T, Ussery DW. RNAmmer: consistent and rapid annotation of ribosomal RNA genes. Nucleic Acids Res. England. 2007;35:3100-8.
33. Altenhoff AM, Škunca N, Glover N, Train C-M, Sueki A, Piližota I, et al. The OMA orthology database in 2015: function predictions, better plant support, synteny view and other improvements. Nucleic Acids Res. 2015;43:D240-9.

34. Rissman Al, Mau B, Biehl BS, Darling AE, Glasner JD, Perna NT. Reordering contigs of draft genomes using the mauve aligner. Bioinformatics. England. 2009:25:2071-3.

35. Noé L, Kucherov G. YASS: enhancing the sensitivity of DNA similarity search. Nucleic Acids Res. 2005;33:W540-3.

36. Trapnell C, Pachter L, Salzberg SL. TopHat: discovering splice junctions with RNA-Seq. Bioinformatics. England. 2009;25:1105-11.

37. Anders S, Pyl PT, Huber W. HTSeq-a python framework to work with highthroughput sequencing data. Bioinformatics. England. 2015;31:166-9.

38. Rhoads A, Au KF. PacBio Sequencing and Its Applications. Genomics. Proteomics Bioinformatics. 2015;13:278-89.

39. Lu H, Giordano F, Ning Z. Oxford Nanopore MinION Sequencing and Genome Assembly. Genomics. Proteomics Bioinformatics 2016;14:265-79.

40. Salzberg SL, Delcher AL, Kasif S, White O. Microbial gene identification using interpolated Markov models. Nucleic Acids Res. England. 1998;26:544-8.

41. Burge C, Karlin S. Prediction of complete gene structures in human genomic DNA. J Mol Biol. England. 1997;268:78-94.

42. Lowe TM, Eddy SR. tRNAscan-SE: a program for improved detection of transfer RNA genes in genomic sequence. Nucleic Acids Res. England. 1997; 25:955-64.

43. Cho S-H, Kim T-S, Lee H-W, Tsuji M, Ishihara C, Kim J-T, et al. Identification of newly isolated Babesia parasites from cattle in Korea by using the Bo-RBCSCID mice. Korean J. Parasitol. 2002:40:33.

44. O'Connor RM, Lane TJ, Stroup SE, Allred DR. Characterization of a variant erythrocyte surface antigen (VESA1) expressed by Babesia bovis during antigenic variation. Mol Biochem Parasitol. Netherlands. 1997;89:259-70.

45. Silva MG, Ueti MW, Norimine J, Florin-Christensen M, Bastos RG, Goff WL, et al. Babesia bovis expresses Bbo-6cys-E, a member of a novel gene family that is homologous to the 6-cys family of plasmodium. Parasitol Int. Netherlands. 2011:60:13-8.

46. Alzan HF, Lau AOT, Knowles DP, Herndon DR, Ueti MW, Scoles GA, et al. Expression of 6-Cys gene Superfamily defines Babesia bovis sexual stage development within Rhipicephalus microplus. PLoS One. United States. 2016:11:e0163791.

47. Kocken CH, Milek RL, Lensen TH, Kaslow DC, Schoenmakers JG, Konings RN. Minimal variation in the transmission-blocking vaccine candidate Pfs48/45 of the human malaria parasite plasmodium falciparum. Mol Biochem Parasitol. Netherlands. 1995;69:115-8.

48. Smith JD, Rowe JA, Higgins MK, Lavstsen T. Malaria's deadly grip: cytoadhesion of plasmodium falciparum-infected erythrocytes. Cell Microbiol. England. 2013;15:1976-83.

49. Horn D. Antigenic variation in African trypanosomes. Mol Biochem Parasitol. Netherlands. 2014;195:123-9.

50. Brownell JE, Zhou J, Ranalli T, Kobayashi R, Edmondson DG, Roth SY, et al. Tetrahymena histone acetyltransferase a: a homolog to yeast Gcn5p linking histone acetylation to gene activation. Cell. United States. 1996;84:843-51.

51. Miao J, Fan Q, Cui L, Li X, Wang H, Ning G, et al. The MYST family histone acetyltransferase regulates gene expression and cell cycle in malaria parasite plasmodium falciparum. Mol Microbiol. England. 2010;78:883-902.

52. Shibayama H, Takai E, Matsumura I, Kouno M, Morii E, Kitamura Y, et al. Identification of a cytokine-induced antiapoptotic molecule anamorsin essential for definitive hematopoiesis. J Exp Med. United States. 2004;199:581-92.

53. Tanimura A, Shibayama H, Hamanaka Y, Fujita N, Ishibashi T, Sudo T, et al. The anti-apoptotic gene Anamorsin is essential for both autonomous and extrinsic regulation of murine fetal liver hematopoiesis. Exp Hematol. Netherlands. 2014:42:410-422.e4.

54. Balaji S, Babu MM, lyer LM, Aravind L. Discovery of the principal specific transcription factors of Apicomplexa and their implication for the evolution of the AP2-integrase DNA binding domains. Nucleic Acids Res. 2005;33: 3994-4006.

55. Yuda M, Iwanaga S, Shigenobu S, Kato T, Kaneko I. Transcription factor AP2$\mathrm{sp}$ and its target genes in malarial sporozoites. Mol Microbiol. England. 2010:75:854-63.

56. Hakimi H, Yamagishi J, Kegawa Y, Kaneko O, Kawazu S-I, Asada M. Establishment of transient and stable transfection systems for Babesia ovata. Parasit Vectors. England. 2016;9:171. 